\title{
Special Issue: Workshop on the Monte Carlo Simulation of Neutron Scattering Instruments MCNSI7
}

\author{
Kim Lefmann ${ }^{\mathrm{a}, *}$ and Jan Šaroun ${ }^{\mathrm{b}}$ \\ ${ }^{a}$ Niels Bohr Institute, Copenhagen University, Copenhagen, Denmark \\ E-mail: lefmann@fys.ku.dk \\ ${ }^{\mathrm{b}}$ Department of Neutron Physics, Nuclear Physics Institute, Řež, Czech Republic \\ E-mail: saroun@ujf.cas.cz.
}

\begin{abstract}
The name MCNSI7 was an acronym for "Monte Carlo Simulations of Neutron Scattering Instruments in FP7". MCNSI7 was a network under the FP7 neutron project NMI3, 2008-2012. The main activities of this network was to hold annual meetings for the neutron simulator community and to arrange a satellite workshop to the ECNS2011 conference in Prague. The workshop attracted 35 participants from 10 countries.
\end{abstract}

The workshop took place at the Faculty of Mathematics and Physics of the Charles University, Prague, whom we thank for providing the venue. The programme included 12 oral presentations and 8 posters. Oral presenters were: Peter Willendrup, Klaus Lieutenant, Jan Saroun, Kim Lefmann, Linda Udby, Alexander Ioffe, Geza Szigmond, Morten Sales, Thilo Seidel, Kaspar Klenø, Nikolaos Tsatpatsaris and Rosaria Mancinelli.

The presenters were given the possibility to submit a manuscript for the workshop proceedings. The present issue of Journal of Neutron Research contains the proceedings of this workshop. The contents of the proceedings give a good flavour of the huge progress in the field of neutron simulation and indicate the level of detail to which simulations are becoming possible. We believe the near future will see detailed simulations being used not only as a tool in instrument construction but also in the actual neutron sciences as a tool to improve understanding of complex data. The MCNSI7 workshop was a small, but important step towards this goal.

\footnotetext{
${ }^{*}$ Corresponding author: Kim Lefmann, Niels Bohr Institute, Copenhagen University, Universitetsparken 5, 2100 Copenhagen, Denmark. E-mail: lefmann@fys.ku.dk.
} 